\title{
FiscalCovid: exercendo o controle social na temática da COVID-19
}

\author{
Cláudio de S. Baptista \\ Laboratório de Sistemas de \\ Informação \\ Universidade Federal de Campina \\ Grande \\ Campina Grande, Paraíba, Brasil \\ baptista@computacao.ufcg.edu.br \\ Mateus Q. Cunha \\ Laboratório de Sistemas de \\ Informação \\ Universidade Federal de Campina \\ Grande \\ Campina Grande, Paraíba, Brasil \\ mateus.cunha@ccc.ufcg.edu.br

\section{Damião Ribeiro de Almeida \\ Laboratório de Sistemas de Informação \\ Universidade Federal de Campina Grande \\ Campina Grande, Paraíba, Brasil damiao@copin.ufcg.edu.br}

\author{
Ana Gabrielle R. Falcão \\ Laboratório de Sistemas de \\ Informação \\ Universidade Federal de Campina \\ Grande \\ Campina Grande, Paraíba, Brasil \\ anagabrielle@copin.ufcg.edu.br
}

\author{
Aillkeen B. Oliveira \\ Laboratório de Sistemas de \\ Informação \\ Universidade Federal de Campina \\ Grande \\ Campina Grande, Paraíba, Brasil \\ aillkeen.oliveira@ccc.ufcg.edu.br
}

\author{
Rich E. C. Ramalho \\ Laboratório de Sistemas de \\ Informação \\ Universidade Federal de Campina \\ Grande \\ Campina Grande, Paraíba, Brasil \\ rich.ramalho@ccc.ufcg.edu.br \\ Júlio H. Rocha \\ Laboratório de Sistemas de \\ Informação \\ Universidade Federal de Campina \\ Grande \\ Campina Grande, Paraíba, Brasil \\ juliorocha@copin.ufcg.edu.br
}

\begin{abstract}
The COVID-19 pandemic had substantial impacts on society, both socially and economically, with devastating impacts on our country. Aiming at assisting on coping with such pandemic and providing relevant information for society on matters such as the portion of the population under quarantine, details on philanthropic institutions and the location of health Units, we developed FiscalCovid. Using geoprocessing and crowdsourcing techniques, this system enables the social control as a support tool for the battle against COVID-19.
\end{abstract}

\section{KEYWORDS}

eCitizenship, Crowdsourcing, Geoprocessing

\section{Introdução}

Nos últimos meses, o mundo tem enfrentado a pandemia da COVID-19, doença que já causou mais de um milhão de mortes e resultou no colapso do sistema de saúde em vários países. A COVID-19 impactou profundamente nossa sociedade, resultando em uma crise de saúde global, com sofrimento social e crise

In: XIX Workshop de Ferramentas e Aplicações (WFA 2020), São Luís, Brasil. Anais Estendidos do Simpósio Brasileiro de Sistemas Multimídia e Web (WebMedia). Porto Alegre: Sociedade Brasileira de Computação, 2020.

(C)2020 SBC - Sociedade Brasileira de Computação.

ISSN: 2596-1683 econômica em todo o mundo. Particularmente no Brasil, a COVID19 tem causado efeitos devastadores, colocando o país como o terceiro país do mundo em número de casos confirmados e o segundo em número de mortes.

Desde o início da pandemia, diversos trabalhos de pesquisa, com foco em Tecnologia da Informação, têm sido desenvolvidos em todo o mundo para facilitar a compreensão e o enfrentamento da evolução desta doença. Normalmente, os trabalhos propostos utilizam diversas tecnologias modernas, principalmente aquelas baseadas em geoprocessamento ([1], [2], [3]) e técnicas de inteligência artificial ([4]; [5]).

Seguindo esta direção, desenvolvemos o FiscalCovid, uma plataforma que usa geoprocessamento e crowdsourcing, cujo objetivo é propiciar à população o efetivo controle social através de denúncias de aglomerações e avaliação de serviços de saúde, bem como informá-la acerca do índice de adesão à quarentena em sua região, fomentando o isolamento social, e o cadastro e consulta às redes de filantropia em sua região, voltadas para donativos para as pessoas mais necessitadas nesta época difícil de pandemia.

$\mathrm{O}$ restante deste artigo está dividido como segue. Na seção 2 , apresentamos a arquitetura do FiscalCovid, seguido, na seção 3 pela descrição de suas principais funcionalidades. Na seção 4, analisamos os impactos sociais e a relevância do FiscalCOVID. Na seção 5, apresentamos os principais resultados obtidos. Finalmente, na seção. 


\section{Arquitetura do FiscalCovid}

O FiscalCovid foi desenvolvido com camadas cliente/servidor, típica de aplicações Web. Esse tipo de aplicação permite um alcance maior ao público, tornando o uso da plataforma mais acessível, pois é possível acessá-la tanto por meio de dispositivos móveis, computadores ou qualquer dispositivo que possa acessar páginas web.

O padrão adotado para desenvolvimento da ferramenta foi o MVC (Model-View-Controller). Na Figura 1 podemos visualizar a arquitetura adotada para o sistema. Essa arquitetura pode ser definida como um conjunto de três camadas: Apresentação: camada responsável por exibir a informação ao usuário; Aplicação: camada responsável pelo gerenciamento dos eventos da aplicação, controlando a parte lógica do sistema; e Dados: camada responsável pelo armazenamento dos dados coletados.

$\mathrm{Na}$ camada de apresentação foram utilizadas as seguintes tecnologias: JavaScript; ReactJS; e OpenLayers. Na camada de aplicação utilizamos Java; Hibernate; Spring Boot, Geoserver e Postgresq1/PostGIS para gestão dos dados.

Além do sistema web, foi desenvolvido um crawler em Python para coletar twitters de usuários com posts relacionados aos sintomas da COVID-19, através da \#fiscalCovid. Esses tweets devem seguir a seguinte estrutura: idade, local da denúncia, algumas respostas sobre sintomas comuns à COVID-19, bem como informações da quarentena do usuário. Após capturar todos esses tweets, é feito um processamento desses dados para obter essas informações e então salvar no banco de dados para serem usados na aplicação do Fiscal Covid.

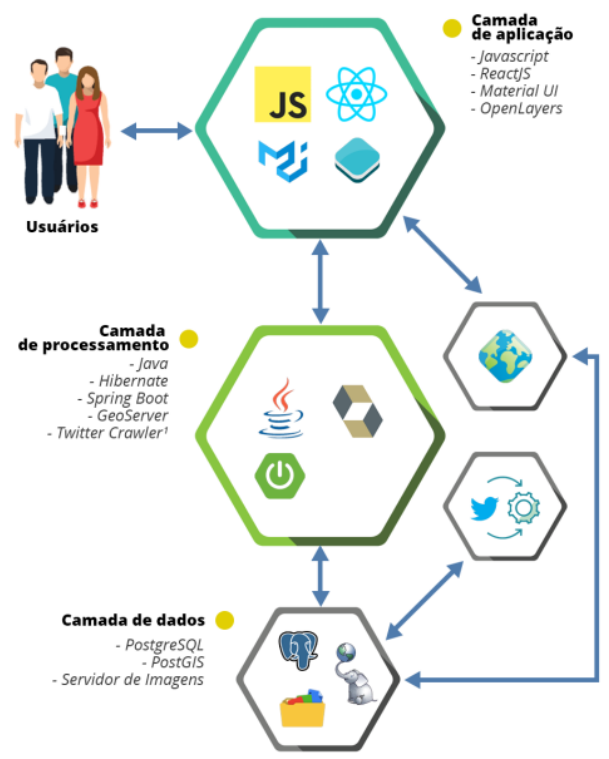

Figura 1: Arquitetura do FiscalCovid

\section{Principais funcionalidades}

Dentre as principais funcionalidades presentes no sistema, podemos iniciar descrevendo o mapa com marcações georreferenciadas de hospitais, Unidades Básicas de Saúde (UBS),
Unidades de Pronto Atendimento (UPA), denúncias de aglomeração e entidades ou projetos filantrópicos (Figura 2).

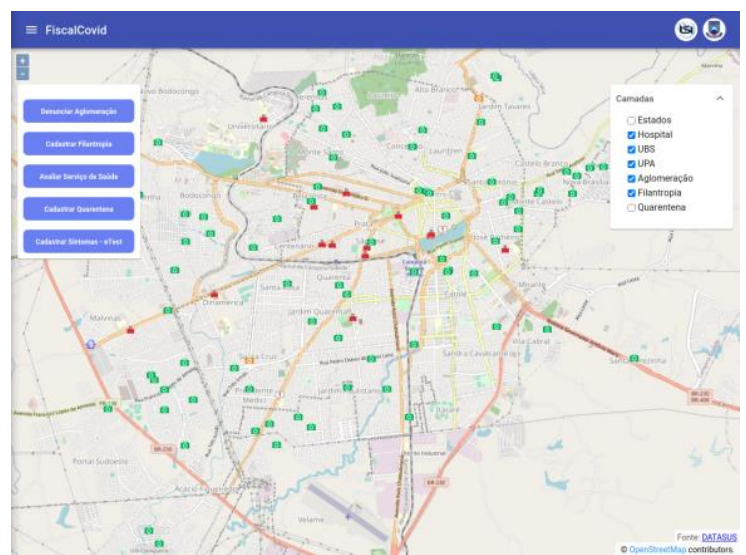

Figura 2: Visualização do mapa do FiscalCovid com as principais camadas selecionadas

Cada item presente no mapa é interativo, que ao serem clicados exibem uma janela de informações com os detalhes e algumas ações que os usuários podem desempenhar no sistema (Figura 3). Os detalhes exibidos para cada tipo de dado são:

1. UBS e UPA: nome da unidade e endereço completo;

2. Hospital: nome da unidade, endereço completo e telefone;

3. Filantropia: nome do projeto ou entidade, descrição, nome do responsável, contato, informa se distribui alimentos, kits de higiene ou realiza campanhas de conscientização e a data de cadastro no sistema;

4. Denúncia de Aglomeração: título, descrição, número de pessoas aglomeradas e data da notícia;

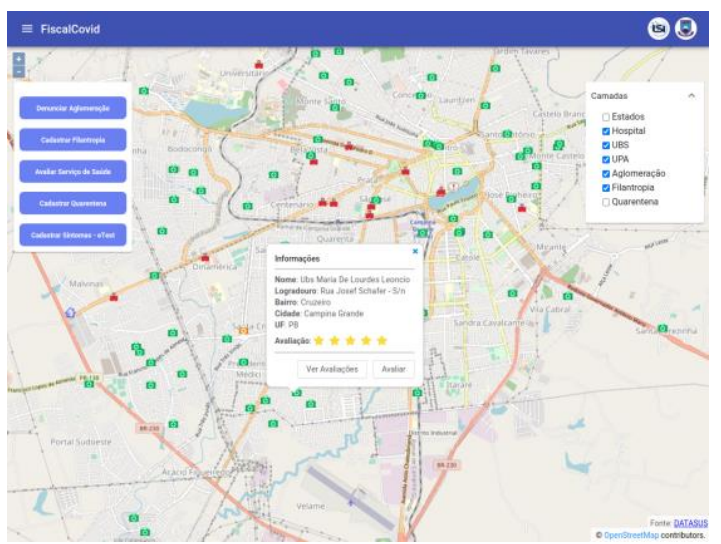

Figura 3: Detalhes exibidos ao clicar numa marcação georreferenciada

As unidades de saúde podem ser avaliadas pelos usuários (Figura 4), atribuindo uma nota e descrevendo o motivo da nota aplicada. Ao exibir os detalhes da unidade de saúde através da funcionalidade de informação do item, o sistema mostra a média 
FiscalCovid: exercendo o controle social na temática da COVID19

das avaliações realizadas, além de permitir visualizar as avaliações feitas por outros usuários. Desta forma, os usuários poderão encontrar as melhores unidades, ou seja, as mais bem avaliadas pela população.

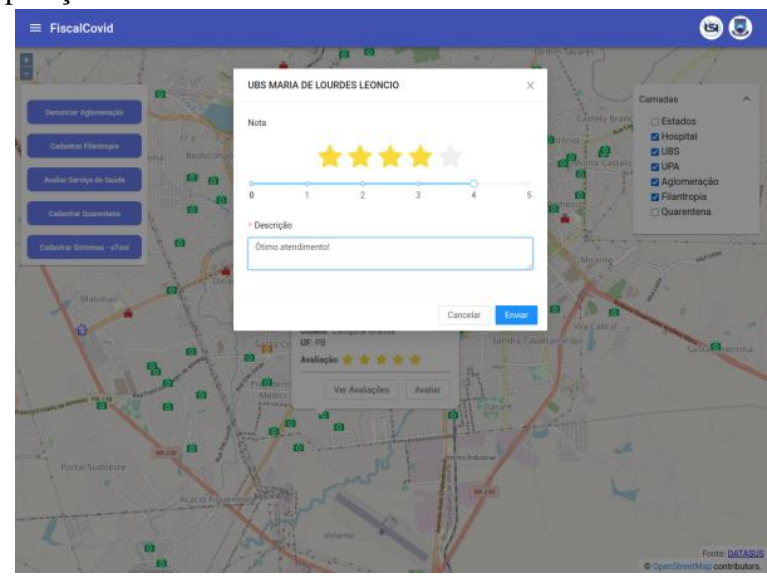

Figura 4: Avaliação de uma unidade de saúde

As denúncias de aglomeração podem ser realizadas pelo próprio cidadão comum. Na Figura 5, ao clicar no botão "Denunciar Aglomeração" e selecionar a localização no mapa, é exibido para o usuário um formulário para inserção das informações de aglomeração. As informações que precisam ser fornecidas pelo usuário são: título da denúncia, descrição e quantidade de pessoas aglomeradas. Ao clicar, no mapa, em uma denúncia já cadastrada, o usuário pode também confirmar a aglomeração e reforçar uma denúncia realizada por outra pessoa.

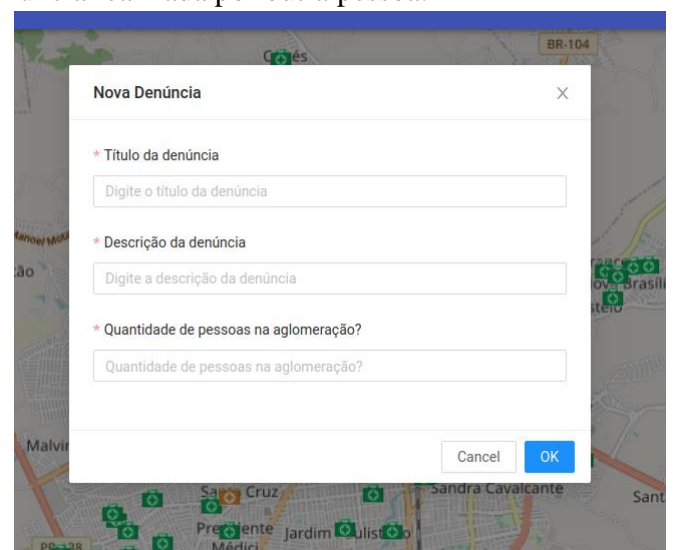

Figura 5: Formulário de denúncia de aglomeração

De forma semelhante, os usuários também podem cadastrar projetos ou instituições filantrópicas no sistema. Ao clicar no botão "Cadastrar Filantropia", o usuário deve clicar no mapa para indicar a localização do novo item a ser cadastrado. Após isso, o sistema exibe um formulário de cadastro para uma nova filantropia (Figura 6). As informações necessárias para o cadastro são: nome da ação, nome do responsável, descrição da ação, contato, permissão para disponibilizar o contato para o público, tipo de atividade
Anais Estendidos do WebMedia'2020, São Luís, Brasil

(distribuição de alimentos, material de higiene, campanha de conscientização ou outros).

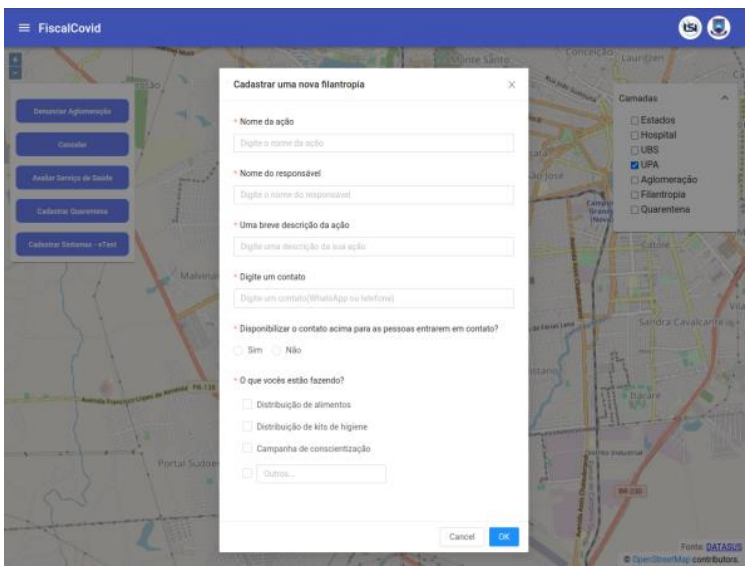

Figura 6: Formulário de cadastro de filantropia

Outra funcionalidade extremamente importante do sistema é o mapa de quarentena, que exibe os dados de adesão de quarentena por cada estado do Brasil (Figura 7). Com isso, a população é capaz de verificar a adesão da região onde vive ao isolamento social.

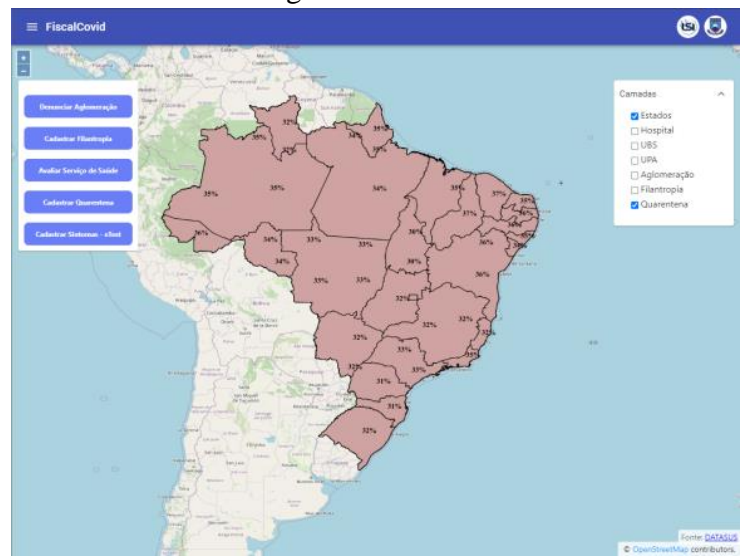

Figura 7: Mapa de quarentena

Além de exibir o mapa de quarentena, o sistema possui o cadastro de quarentena (Figura 8). Esta funcionalidade consiste no cadastro de informações sobre a adesão da quarentena na residência do usuário, contando com informações como número de pessoas residentes e quantas delas estão de fato aderindo a quarentena. $\mathrm{O}$ intuito é sumarizar esses dados por município e obter o percentual de adesão/não adesão ao isolamento social.

Como forma de reduzir a subnotificação, foi desenvolvida a funcionalidade chamada "eTeste de Sintomas" (Figura 9). Neste teste digital é possível informar dados sobre sintomas que possivelmente o próprio usuário, algum familiar ou conhecido possam estar apresentando. Além da localização e email para contato, o usuário precisa especificar o tipo de sintoma apresentado por ele ou conhecido, tais como, febre alta, dificuldade para respirar ou dor de garganta. 
Atualmente, o sistema está limitado para o cenário criado com a pandemia da COVID-19, porém ele poderá, em trabalhos futuros, ser estendido para abranger situações ocasionadas por outras temáticas, com controles mais específicos e filtros diferenciados. Outra limitação do sistema, que ainda não foi tratada, seria uma visualização temporal das denúncias de aglomeração, visto que estes são eventos passageiros.

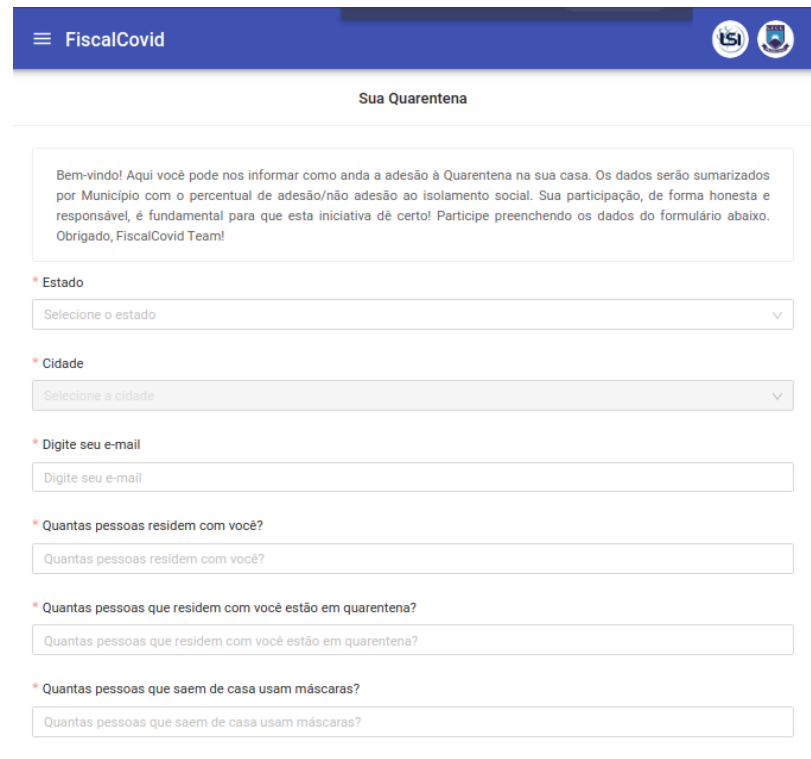

Figura 8: Formulário de cadastro de quarentena

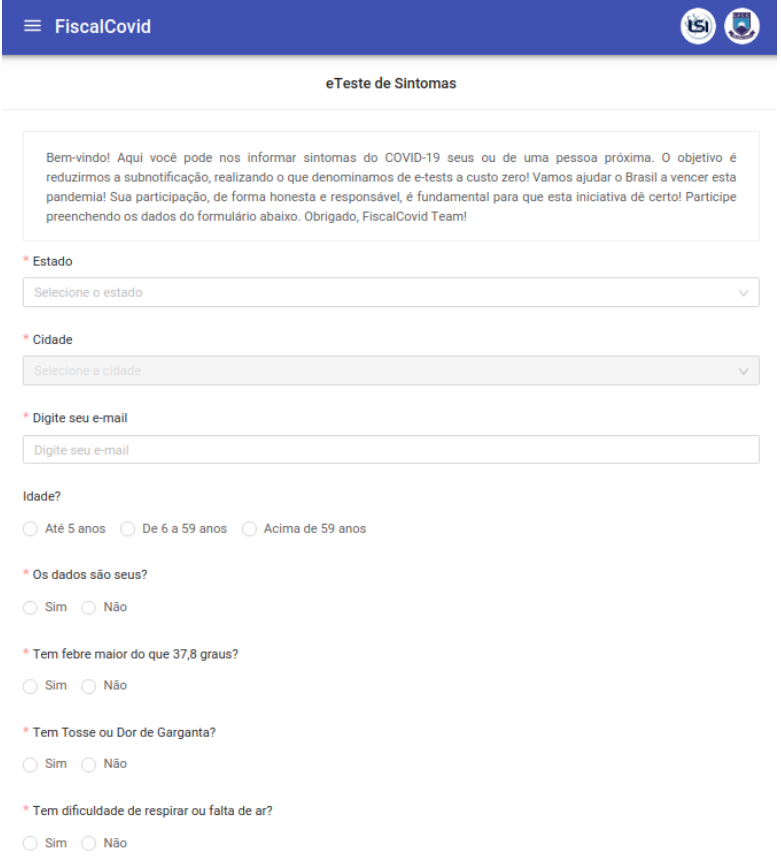

Figura 9: Formulário do eTeste de Sintomas

\section{Relevância, Impacto Social e Executabilidade}

A sociedade moderna prescinde de ferramentas de e-participatory [6], com as quais os cidadãos podem aferir, avaliar, consultar, encontrar e comentar sobre diversos serviços prestados à sociedade pelo setor público nas diversas esferas Federal, Estadual e Municipal. Com isso, o controle social é exercido plenamente pelos cidadãos que com seus impostos sustentam tais serviços.

Outrossim, os órgãos de controle: Tribunais de Contas: da União, Estados e Municípios e Controladorias Federais, Estaduais e Municipais, podem se valer das informações provindas da sociedade em plataformas de crowdsourcing para melhor fiscalizar as ações dos gestores públicos, particularmente na área de saúde, evitando o desperdício e uso inadequado dos recursos públicos, bem como combatendo os atos de corrupção. Por fim, entidades não governamentais como ONGs e OSCIPs e a própria imprensa também podem, utilizando-se das avaliações e queixas dos usuários realizar ações em prol da melhoria dos serviços prestados, bem como melhor avaliar cenários de adesão à quarentena e aglomerações.

O uso de indicadores (KPI) para gestão moderna e eficaz é imprescindível. Particularmente, no tocante à pandemia, podem ser utilizados diversos destes indicadores relacionados à atenção básica, custo per capta na função saúde, taxa de unidades móveis de atendimento por 100 mil habitantes, número de médicos / enfermeiros /odontólogos / fisioterapeutas por 100 mil habitantes para mensurar o SROI, o retorno do investimento feito na saúde versus a qualidade dos serviços prestados. O Instituto Rui Barbosa tem usado o IEGM - Índice de Efetividade da Gestão Municipal (IEGM), que é um indicador (KPI) que mede o grau de aderência da gestão municipal a determinados processos e controles em sete áreas: educação, saúde, gestão fiscal, planejamento, meio ambiente, defesa civil e governança em tecnologia da informação. Particularmente, no tocante à gestão da pandemia, pode-se o utilizar o IEGM - i-Saúde para mensurar o SROI. O i-Saúde é o Índice Municipal da Saúde, utilizando de diversos parâmetros relacionados à Atenção Básica, Cobertura e ação do Programa Saúde da Família, atuação do Conselho Municipal da Saúde, assiduidade dos médicos e profissionais de saúde, atendimento à população para tratamento de doenças realização de exames, controle de estoque de insumos, distribuição de medicamentos, cobertura das campanhas de vacinação e de orientação à população. Perceba que estes parâmetros do i-Saúde podem ser obtidos da própria população através de uma ferramenta como o FiscalCovid.

No que tange à executabilidade do projeto, reputamos o FiscalCovid como de fácil implantação, uma vez que é preciso apenas hospedar o sistema numa plataforma em nuvem. Todo o software utilizado é software livre, sem TCO (Total Cost of Ownership), sem risco associado. O único desafio que enfrentamos é convencer uma Prefeitura da importância da e-cidadania e do controle social, pois certamente o FiscalCovid pode vir a expor algum lado negativo na gestão de saúde de um governo. No entanto, entendemos que este desafio é um lado bom, pois entendemos que o gestor sério e competente irá querer ouvir da população os seus problemas e enfrentá-los o mais cedo possível, dando resolubilidade aos problemas. Outrossim, órgãos de controle bem 
FiscalCovid: exercendo o controle social na temática da COVID19

como entidades não governamentais também poderiam absorver o FiscalCovid como plataforma de controle social.

Quanto à usabilidade, o sistema leva em consideração todo o conhecimento adquirido no estudo e validação do Crowd4City, plataforma similar que também faz uso de crowdoursing e técnicas de geoprocessamento em busca do efetivo controle social [7].

\section{Resultados}

O projeto está em fase de desenvolvimento e atualmente buscamos órgãos parceiros que possam se interessar pela plataforma FiscalCovid para que possamos lançá-la para uso efetivo. Há um protótipo em funcionamento instalado em um servidor do LSI Laboratório de Sistemas de Informação da UFCG no endereço: http://fiscalcovid.lsi.ufcg.edu.br/. Atualmente, temos algumas entidades filantrópicas cadastradas, que obtivemos do Instituto Marielle Franco, e algumas denúncias de aglomerações. Também as informações do mapa de quarentena são colhidas diariamente do site: https://mapabrasileirodacovid.inloco.com.br/

\section{Cronograma}

Nosso roadmap inclui as seguintes atividades:

1. Aperfeiçoamento da coleta de dados a partir de redes sociais (Twitter), com implantação de algoritmos de análise de sentimentos e análise de emoção - 3 meses;

2. Introdução de indicadores de saúde para mensurar o SROI; 6 meses e;

3. Uso de algoritmos de análise de sentimentos e de emoção nos comentários realizados na plataforma - 3 meses.

\section{ACKNOWLEDGMENTS}

Os autores agradecem ao CNPQ - Conselho Nacional de Desenvolvimento Científico e Tecnológico por financiar parcialmente esta pesquisa.

\section{REFERENCES}

[1] Z. Peng, R. Wang, L. Liu, H. Wu. Exploring Urban Spatial Features of COVID-19 Transmission in Wuhan Based on Social Media Data. ISPRS Int. J. Geo Inf. 9(6): $402(2020)$

[2] U. Qazi, M. Imran, F. Ofli. GeoCoV19: a dataset of hundreds of millions of multilingual COVID-19 tweets with location information. ACM SIGSPATIAL Special 12(1): 6-15 (2020)

[3] A. Züfle. Introduction to this special issue: Modeling and understanding the spread of COVID-19: (part I). ACM SIGSPATIAL Special 12(1): 1-2 (2020)

[4] W. Naudé. Artificial intelligence vs COVID-19: limitations, constraints and pitfalls. AI Soc. 35(3): 761-765 (2020)

[5] A. A. Hussain, O. Bouachir, F. Al-Turjman, M. Aloqaily. AI Techniques for COVID-19. IEEE Access 8: 128776-128795 (2020)

[6] T. R. Coelho, M. A. Cunha, M. Pozzebon. eParticipation and the policy cycle: Designing a research agenda. DG.O 2017: 368-376

[7] A. G. R. Falcão, P. F. Wanderley, T. H. S. Leite, C. S. Bapista, J. E. R. de Queiroz, M. G. de Oliveira, J. H. Rocha. Crowdsourcing Urban Issues in Smart Cities: a Usability Assessment of the Crowd4City System. 10.1007/978-3-030-275235_11(2019)

\section{Currículos}

Cláudio de Souza Baptista, é Professor Titular do Departamento de Sistemas e Computação da Universidade Federal de Campina Grande (UFCG), onde Coordena o Laboratório de Sistemas de Informação (LSI). Atua no Doutorado, Mestrado e Bacharelado em Ciência da Computação. É pesquisador DTI-2A do CNPQ. Publicou mais de 100 artigos em periódicos e congressos nacionais e internacionais. Suas áreas de interesse incluem: multimídia, banco de dados, geoprocessamento e processamento de linguagem natural.

http://lattes.cnpq.br/0104124422364023

Ana Gabrielle Ramos Falcão, é Graduada e Mestre em Ciência da Computação pela Universidade Federal de Campina Grande. Atualmente trabalha com Pesquisa e Desenvolvimento no Laboratório de Sistemas de Informação (LSI/UFCG), com experiência nas áreas de Banco de Dados, Sistemas de Informação e Sistemas de Informação Geográficos.

http://lattes.cnpq.br/4654742780714984

Rich Elton Carvalho Ramalho, é graduando em Ciência da Computação na Universidade Federal de Campina Grande, se encontra no $6^{\circ}$ período. Atua em projetos de pesquisa e de desenvolvimento no Laboratório de Sistemas de Informação (LSI). As suas áreas de interesse incluem: desenvolvimento web, processamento de linguagem natural e machine learning.

http://lattes.cnpq.br/7686727918516258

Mateus Queiroz Cunha é Técnico em Manutenção e Suporte em Informática pelo Instituto Federal de Ciência e Tecnologia da Paraíba e Graduando em Ciência da Computação pela Universidade Federal de Campina Grande. Atua como desenvolvedor e pesquisador no Laboratório de Sistemas de Informação (LSI/UFCG).

http://lattes.cnpq.br/6398825826705311

Aillkeen Bezerra de Oliveira, é Graduado em Ciência da Computação pela Universidade Federal de Campina Grande (UFCG). Atua como desenvolvedor/pesquisador no Laboratório de Sistemas de Informação (LSI/UFCG). Tem experiência na área de Banco de Dados, Sistemas de Informação, Inteligência Artificial e Sistemas para dispositivos móveis.

http://lattes.cnpq.br/5681431499623786

Júlio Henrique Rocha é graduado e Mestre no curso de Ciência da Computação pela Universidade Federal de Campina Grande Campus I. Na graduação participou de projetos de Iniciação Científica. Atualmente participa como Analista de Sistemas Sênior no Laboratório de Sistemas de Informação (LSI/UFCG). Tem experiência na grande área de Ciência da Computação, com ênfase em Sistemas de Informação, Sistemas de Informação Geográfica e Banco de Dados Espacial.

http://lattes.cnpq.br/8217213614133628

Damião Ribeiro de Almeida é graduado e Mestre no curso de Ciência da Computação pela Universidade Federal de Campina Grande - UFCG. Atua como pesquisador e desenvolvedor no Laboratório de Sistemas de Informação (LSI/UFCG) com ênfase nas áreas de Business Intelligence, Banco de Dados Espaciais e Sistemas de Informações Geográficas.

http://lattes.cnpq.br/4070628413303784 